\title{
A stochastic diffusion process for Lochner's generalized Dirichlet distribution
}

\author{
LA-UR 13-21573 \\ Accepted for publication in Journal of Mathematical Physics, September 12, 2013 \\ J. Bakosi, J.R. Ristorcelli \\ \{jbakosi, jrrj\}@lanl.gov \\ Los Alamos National Laboratory, Los Alamos, NM 87545, USA
}

\begin{abstract}
The method of potential solutions of Fokker-Planck equations is used to develop a transport equation for the joint probability of $N$ stochastic variables with Lochner's generalized Dirichlet distribution [1] as its asymptotic solution. Individual samples of a discrete ensemble, obtained from the system of stochastic differential equations, equivalent to the Fokker-Planck equation developed here, satisfy a unit-sum constraint at all times and ensure a bounded sample space, similarly to the process developed in [2] for the Dirichlet distribution. Consequently, the generalized Dirichlet diffusion process may be used to represent realizations of a fluctuating ensemble of $N$ variables subject to a conservation principle. Compared to the Dirichlet distribution and process, the additional parameters of the generalized Dirichlet distribution allow a more general class of physical processes to be modeled with a more general covariance matrix.
\end{abstract}

Keywords: Fokker-Planck equation; Stochastic diffusion; Generalized Dirichlet distribution

\section{Introduction}

We develop a Fokker-Planck equation whose statistically stationary solution (i.e. invariant) is Lochner's generalized Dirichlet distribution [1, 3, 4].

The (standard) Dirichlet distribution [5, 6, 7] has been used to represent a set of non-negative fluctuating variables subject to a unit-sum requirement in a variety of fields, including evolutionary theory [8], Bayesian statistics [9], geology [10, 11], forensics [12], econometrics [13], turbulent combustion [14], and population biology [15]. Following the method of potential solutions, applied in [2], we derive a system of coupled stochastic differential equations (SDE) whose (Wiener-process) diffusion terms are nonlinearly coupled and whose invariant is Lochner's generalized Dirichlet distribution.

The standard Dirichlet distribution can only represent non-positive covariances [6], which limits its application to a specific class of processes. The stochastic system whose invariant is the generalized Dirichlet distribution allows for a more general class of physical processes with a more general covariance matrix. The process may be stationary or non-stationary, not limited to non-positive covariances, and satisfies the unit-sum requirement at all times, necessary for variables that obey a conservation principle.

\section{Preview of results}

The generalized Dirichlet distribution for a set of scalars $0 \leq Y_{i}, i=1, \ldots, K, \sum_{i=1}^{K} Y_{i} \leq 1$, and parameters, $\alpha_{i}>0, \beta_{i}>0$, as given by Lochner [1] reads

$$
\mathscr{G}(\mathbf{Y}, \boldsymbol{\alpha}, \boldsymbol{\beta})=\prod_{i=1}^{K} \frac{\Gamma\left(\alpha_{i}+\beta_{i}\right)}{\Gamma\left(\alpha_{i}\right) \Gamma\left(\beta_{i}\right)} Y_{i}^{\alpha_{i}-1} \mathcal{Y}_{i}^{\gamma_{i}} \quad \text { with } \quad \mathcal{Y}_{i}=1-\sum_{k=1}^{i} Y_{k},
$$


and $\gamma_{i}=\beta_{i}-\alpha_{i+1}-\beta_{i+1}$ for $i=1, \ldots, K-1$, and $\gamma_{K}=\beta_{K}-1$. Here $\Gamma(\cdot)$ denotes the gamma function. We derive the stochastic diffusion process, governing the scalars, $Y_{i}$,

$$
\begin{array}{r}
\mathrm{d} Y_{i}(t)=\frac{\mathcal{U}_{i}}{2}\left\{b_{i}\left[S_{i} \mathcal{Y}_{K}-\left(1-S_{i}\right) Y_{i}\right]+Y_{i} \mathcal{Y}_{K} \sum_{j=i}^{K-1} \frac{c_{i j}}{\mathcal{Y}_{j}}\right\} \mathrm{d} t+\sqrt{\kappa_{i} Y_{i} \mathcal{Y}_{K} \mathcal{U}_{i}} \mathrm{~d} W_{i}(t), \\
i=1, \ldots, K,
\end{array}
$$

where $\mathrm{d} W_{i}(t)$ is an isotropic vector-valued Wiener process with independent increments [16] and $\mathcal{U}_{i}=\prod_{j=1}^{K-i} \mathcal{Y}_{K-j}^{-1}$. We show that the statistically stationary solution of the coupled system of nonlinear stochastic differential equations in (2) is the generalized Dirichlet distribution, Eq. (1), provided the coefficients, $b_{i}>0, \kappa_{i}>0,0<S_{i}<1$, and $c_{i j}$, with $c_{i j}=0$ for $i>j, i, j=1, \ldots, K-1$, satisfy

$$
\begin{aligned}
\alpha_{i} & =\frac{b_{i}}{\kappa_{i}} S_{i}, \quad i=1, \ldots, K, \\
1-\gamma_{i} & =\frac{c_{1 i}}{\kappa_{1}}=\cdots=\frac{c_{i i}}{\kappa_{i}}, \quad i=1, \ldots, K-1, \\
1+\gamma_{K} & =\frac{b_{1}}{\kappa_{1}}\left(1-S_{1}\right)=\cdots=\frac{b_{K}}{\kappa_{K}}\left(1-S_{K}\right) .
\end{aligned}
$$

The restriction on the coefficients ensure reflection towards the interior of the sample space, which together with the specification $Y_{N}=\mathcal{Y}_{K}$ ensures

$$
\sum_{i=1}^{N} Y_{i}=1
$$

Indeed, if for example $Y_{1}=0$, the diffusion in Eq. (2) is zero and the drift is strictly positive, while if $Y_{1}=1$, the diffusion is zero (as $\mathcal{Y}_{K} \mathcal{U}_{1} \rightarrow 0$ ) and the drift is strictly negative.

\section{Development of the diffusion process}

The diffusion process, Eq. (2), is developed by the method of potential solutions. The steps below closely follow the methodology introduced in [2], used to derive a diffusion process for the Dirichlet distribution.

We start from the Itô diffusion process [16] for the stochastic vector, $Y_{i}$,

$$
\mathrm{d} Y_{i}(t)=a_{i}(\mathbf{Y}) \mathrm{d} t+\sum_{j=1}^{K} b_{i j}(\mathbf{Y}) \mathrm{d} W_{j}(t), \quad i=1, \ldots, K,
$$

with drift, $a_{i}(\mathbf{Y})$, diffusion, $b_{i j}(\mathbf{Y})$, and the isotropic vector-valued Wiener process, $\mathrm{d} W_{j}(t)$. Using standard methods given in [16] the equivalent Fokker-Planck equation governing the joint probability, $\mathscr{F}(\mathbf{Y}, t)$, derived from Eq. (7), is

$$
\frac{\partial \mathscr{F}}{\partial t}=-\sum_{i=1}^{K} \frac{\partial}{\partial Y_{i}}\left[a_{i}(\mathbf{Y}) \mathscr{F}\right]+\frac{1}{2} \sum_{i=1}^{K} \sum_{j=1}^{K} \frac{\partial^{2}}{\partial Y_{i} \partial Y_{j}}\left[B_{i j}(\mathbf{Y}) \mathscr{F}\right], \quad B_{i j}=\sum_{k=1}^{K} b_{i k} b_{k j} .
$$

As the drift and diffusion coefficients are time-homogeneous, $a_{i}(\mathbf{Y}, t)=a_{i}(\mathbf{Y})$ and $B_{i j}(\mathbf{Y}, t)=$ $B_{i j}(\mathbf{Y})$, Eq. (7) is a statistically stationary process and the solution of Eq. (8) converges to a stationary distribution [16], Sec. 6.2.2. Our task is to specify the functional forms of $a_{i}(\mathbf{Y})$ and $b_{i j}(\mathbf{Y})$ so that the stationary solution of Eq. (8) is $\mathscr{G}(\mathbf{Y})$, defined by Eq. (1). 
A potential solution of Eq. (8) exists if

$$
\frac{\partial \ln \mathscr{F}}{\partial Y_{j}}=\sum_{i=1}^{K} B_{i j}^{-1}\left(2 a_{i}-\sum_{k=1}^{K} \frac{\partial B_{i k}}{\partial Y_{k}}\right) \equiv-\frac{\partial \phi}{\partial Y_{j}}, \quad j=1, \ldots, K,
$$

is satisfied, [16] Sec. 6.2.2. Since the left hand side of Eq. (9) is a gradient, the expression on the right must also be a gradient and can therefore be obtained from a scalar potential denoted by $\phi(\mathbf{Y})$. This puts a constraint on the possible choices of $a_{i}$ and $B_{i j}$ and on the potential, as $\phi, i j=\phi,{ }_{j i}$ must also be satisfied. The potential solution is

$$
\mathscr{F}(\mathbf{Y})=\exp [-\phi(\mathbf{Y})]
$$

Now functional forms of $a_{i}(\mathbf{Y})$ and $B_{i j}(\mathbf{Y})$ that satisfy Eq. $(9)$, with $\mathscr{F}(\mathbf{Y}) \equiv \mathscr{G}(\mathbf{Y})$ are sought. The mathematical constraints on the specification of $a_{i}$ and $B_{i j}$ are as follows:

1. $B_{i j}$ must be symmetric positive semi-definite. This is to ensure that

- the square-root of $B_{i j}$ (e.g. the Cholesky-decomposition, $b_{i j}$ ) exists, required by the correspondence of the stochastic equation (7) and the Fokker-Planck equation (8),

- Eq. (7) represents a diffusion, and

- $\operatorname{det}\left(B_{i j}\right) \neq 0$, required by the existence of the inverse in Eq. (9).

2. For a potential solution to exist Eq. (9) must be satisfied.

With $\mathscr{F}(\mathbf{Y}) \equiv \mathscr{G}(\mathbf{Y})$ Eq. (10) shows that the scalar potential must be

$$
-\phi(\mathbf{Y})=\sum_{i=1}^{K}\left(\alpha_{i}-1\right) \ln Y_{i}+\sum_{i=1}^{K} \gamma_{i} \ln \mathcal{Y}_{i}
$$

It is straightforward to verify that the specifications

$$
\begin{aligned}
a_{i}(\mathbf{Y}) & =\frac{\mathcal{U}_{i}}{2}\left\{b_{i}\left[S_{i} \mathcal{Y}_{K}-\left(1-S_{i}\right) Y_{i}\right]+Y_{i} \mathcal{Y}_{K} \sum_{j=i}^{K-1} \frac{c_{i j}}{\mathcal{Y}_{j}}\right\}, \\
B_{i j}(\mathbf{Y}) & = \begin{cases}\kappa_{i} Y_{i} \mathcal{Y}_{K} \mathcal{U}_{i} & \text { for } \quad i=j, \\
0 & \text { for } \quad i \neq j,\end{cases}
\end{aligned}
$$

satisfy the above mathematical constraints, 1. and 2. Here $\mathcal{U}_{i}=\prod_{j=1}^{K-i} \mathcal{Y}_{K-j}^{-1}$, where an empty product is assumed to be unity, while an empty sum is zero. In addition to the coefficients $b_{i}>0$, $\kappa_{i}>0$, and $0<S_{i}<1$, governing the Dirichlet diffusion process [2], the drift now has the additional (not all independent) ones, denoted by $c_{i j}$, with $c_{i j}=0$ for $i>j, i, j=1, \ldots, K-1$.

Substituting Eqs. (11-13) into Eq. (9) yields a system with the same functions on both sides with different coefficients, yielding the correspondence between the parameters of the generalized Dirichlet distribution, Eq. (1), and the Fokker-Planck equation (8) with Eqs. (12-13) as

$$
\begin{aligned}
\alpha_{i} & =\frac{b_{i}}{\kappa_{i}} S_{i}, \quad i=1, \ldots, K, \\
1-\gamma_{i} & =\frac{c_{1 i}}{\kappa_{1}}=\cdots=\frac{c_{i i}}{\kappa_{i}}, \quad i=1, \ldots, K-1, \\
1+\gamma_{K} & =\frac{b_{1}}{\kappa_{1}}\left(1-S_{1}\right)=\cdots=\frac{b_{K}}{\kappa_{K}}\left(1-S_{K}\right) .
\end{aligned}
$$


The above result is arrived at inductively based on the special case of $K=3$ in Appendix A. If Eqs. (14-16) hold, the stationary solution of the Fokker-Planck equation (8) with drift (12) and diffusion (13) is the generalized Dirichlet distribution, Eq. (1). The same methodology was applied to the Dirichlet case in [2]. Eqs. (14-16) specify the correspondence between the coefficients of the stochastic system (7) with drift (12) and diffusion (13) and the generalized Dirichlet distribution, Eq. (1). With $\gamma_{i}=\beta_{i}-\alpha_{i+1}-\beta_{i+1}, i=1, \ldots, K-1$, and $\gamma_{K}=\beta_{K}-1$, the correspondence between $\left(\alpha_{i}, \beta_{i}\right)$ and $\left(b_{i}, S_{i}, \kappa_{i}, c_{i j}\right)$ is also complete. Note that Eqs. (12-13) are one possible way of specifying drift and diffusion to arrive at a generalized Dirichlet distribution; other functional forms may be possible. It is straightforward to verify, that setting $c_{1 i} / \kappa_{i}=\cdots=c_{i i} / \kappa_{i}=1$ for $i=1, \ldots, K-1$, i.e., $\gamma_{1}=\cdots=\gamma_{K-1}=0$, in Eqs. (12) and (13) yields the same system in Eq. (9) as with $a_{i}$ and $B_{i j}$ specified for the (standard) Dirichlet distribution, see Appendix A for $K=3$. The shape of the generalized Dirichlet distribution, Eq. (1), is determined by the $2 K$ coefficients, $\alpha_{i}, \beta_{i}$. Eqs. (14-16) show that in the stochastic system, different combinations of $b_{i}$, $S_{i}, \kappa_{i}$, and $c_{i j}$ may yield the same $\alpha_{i}, \beta_{i}$ and that not all of $b_{i}, S_{i}, \kappa_{i}$, and $c_{i j}$ may be chosen independently to make the invariant generalized Dirichlet. In other words, a unique set of SDE coefficients always corresponds to a unique set of distribution parameters, but the converse is not true: a set of distribution parameters do not uniquely determine all the SDE coefficients, for a given specific asymptotic generalized Dirichlet distribution.

\section{Properties of Dirichlet distributions}

It is useful to show how the generalized Dirichlet distribution, Eq. (1), reduces to standard Dirichlet, and their univariate case, the beta distribution.

\subsection{Density functions}

Setting $\gamma_{1}=\cdots=\gamma_{K-1}=0$ in Eq. (1) yields the (standard) Dirichlet distribution

$$
\mathscr{D}(\mathbf{Y}, \boldsymbol{\omega})=\frac{\Gamma\left(\sum_{i=1}^{N} \omega_{i}\right)}{\prod_{i=1}^{N} \Gamma\left(\omega_{i}\right)} \prod_{i=1}^{N} Y_{i}^{\omega_{i}-1}
$$

with $\omega_{i}=\alpha_{i}, i=1, \ldots, K=N-1, \omega_{N}=\beta_{K}$, and $Y_{N}=1-\sum_{j=1}^{K} Y_{j}$. In the univariate case, $K=N-1=1, \mathbf{Y}=\left(Y_{1}, Y_{2}\right)=(Y, 1-Y)$, both $\mathscr{G}$ and $\mathscr{D}$ yield the beta distribution

$$
\mathscr{B}(Y, \alpha, \beta)=\frac{\Gamma(\alpha+\beta)}{\Gamma(\alpha) \Gamma(\beta)} Y^{\alpha-1}(1-Y)^{\beta-1},
$$

with $\omega_{1}=\alpha$ and $\omega_{2}=\beta$.

$\mathscr{G}, \mathscr{D}$, and $\mathscr{B}$ are zero outside the $K$-dimensional generalized triangle; the sample spaces are bounded. Compared to $\mathscr{D}$, there are $K-1$ additional parameters in $\mathscr{G}$ for a set of $K$ scalars.

\subsection{Moments}

All moments of the generalized Dirichlet distribution, Eq. (1), can be obtained from $\alpha_{i}$ and $\beta_{i}$ of which the first two are $[3,4]$

$$
\begin{aligned}
\left\langle Y_{i}\right\rangle=\int Y_{i} \mathscr{G}(\mathbf{Y}) \mathrm{d} \mathbf{Y}=\frac{\alpha_{i}}{\alpha_{i}+\beta_{i}} \prod_{j=1}^{i-1} \frac{\beta_{j}}{\alpha_{j}+\beta_{j}}, \\
\left\langle y_{i} y_{j}\right\rangle=\left\langle\left(Y_{i}-\left\langle Y_{i}\right\rangle\right)\left(Y_{j}-\left\langle Y_{j}\right\rangle\right)\right\rangle= \begin{cases}\left\langle Y_{i}\right\rangle\left(\frac{\alpha_{i}+1}{\alpha_{i}+\beta_{i}+1} M_{i-1}-\left\langle Y_{i}\right\rangle\right) & \text { for } \quad i=j, \\
\left\langle Y_{j}\right\rangle\left(\frac{\alpha_{i}}{\alpha_{i}+\beta_{i}+1} M_{i-1}-\left\langle Y_{i}\right\rangle\right) & \text { for } i \neq j,\end{cases}
\end{aligned}
$$




$$
i, j=1, \ldots, K
$$

where $M_{i-1}=\prod_{k=1}^{i-1}\left(\beta_{k}+1\right) /\left(\alpha_{k}+\beta_{k}+1\right)$. Setting $\gamma_{1}=\cdots=\gamma_{K-1}=0$, with $\omega_{i}=\alpha_{i}$, $i=1, \ldots, K=N-1, \omega_{N}=\beta_{K}$, in Eqs. (19-20) reduces to the first two moments of the Dirichlet distribution,

$$
\begin{aligned}
\left\langle Y_{i}\right\rangle & =\frac{\omega_{i}}{\omega}, \\
\left\langle y_{i} y_{j}\right\rangle & =\left\{\begin{array}{lr}
\frac{\omega_{i}\left(\omega-\omega_{i}\right)}{\omega^{2}(\omega+1)} & \text { for } i=j, \\
\frac{-\omega_{i} \omega_{j}}{\omega^{2}(\omega+1)} & \text { for } i \neq j, \\
i, j=1, \ldots, K,
\end{array}\right.
\end{aligned}
$$

where $\omega=\sum_{j=1}^{N} \omega_{j}$. Eq. (20) shows that in the generalized Dirichlet distribution $Y_{1}$ is always negatively correlated with the other scalars. However, $Y_{j}$ and $Y_{m}$ can be positively correlated for $j, m>1$, see also [1]. According to Wong [4], "If there exists some $m>j$ such that $Y_{j}$ and $Y_{m}$ are positively (negatively) correlated, then $Y_{j}$ will be positively (negatively) correlated with $Y_{n}$ for all $n>j$." This can be seen from Eq. (20): the sign of $\left\langle y_{m} y_{j}\right\rangle$ is independent of $j$, so the sign of $\left\langle y_{m} y_{j}\right\rangle, m>j$ will imply the signs of all $\left\langle y_{n} y_{j}\right\rangle, n>j$. This is in contrast with the Dirichlet distribution, Eq. (17), whose covariances are always non-positive as can be seen from Eq. (22).

In the univariate case, $K=N-1=1, \mathbf{Y}=\left(Y_{1}, Y_{2}\right)=(Y, 1-Y)$, the first two moments of both the generalized and the standard Dirichlet distributions, Eqs. (19-20) and Eqs. (21-22), respectively, reduce to the moments of the beta distribution, with $\omega_{1}=\alpha$ and $\omega_{2}=\beta$,

$$
\begin{aligned}
\langle Y\rangle & =\frac{\alpha}{\alpha+\beta}, \\
\left\langle y^{2}\right\rangle & =\frac{\alpha \beta}{(\alpha+\beta)^{2}(\alpha+\beta+1)} .
\end{aligned}
$$

\section{Relation to other diffusion processes}

It also useful to relate the generalized Dirichlet process, Eq. (2), to other multivariate diffusion processes with linear drift and quadratic diffusion.

Setting $c_{1 i} / \kappa_{i}=\cdots=c_{i i} / \kappa_{i}=1$ for $i=1, \ldots, K-1$, in Eq. (2) yields

$$
\mathrm{d} Y_{i}(t)=\frac{b_{i}}{2}\left[S_{i} Y_{N}-\left(1-S_{i}\right) Y_{i}\right] \mathrm{d} t+\sqrt{\kappa_{i} Y_{i} Y_{N}} \mathrm{~d} W_{i}(t), \quad i=1, \ldots, K=N-1,
$$

with $Y_{N}=1-\sum_{j=1}^{N-1} Y_{j}$ whose invariant is the (standard) Dirichlet distribution, Eq. (17). Eq. (25) is discussed in [2]. Another diffusion process whose invariant is also Dirichlet is the multivariate Wright-Fisher process [15],

$$
\mathrm{d} Y_{i}(t)=\frac{1}{2}\left(\omega_{i}-\omega Y_{i}\right) \mathrm{d} t+\sum_{j=1}^{K} \sqrt{Y_{i}\left(\delta_{i j}-Y_{j}\right)} \mathrm{d} W_{i j}(t), \quad i=1, \ldots, K=N-1,
$$

where $\delta_{i j}$ is Kronecker's delta. Another process similar to Eqs. (2), (25), and (26) is the multivariate Jacobi process, used in econometrics,

$$
\mathrm{d} Y_{i}(t)=a\left(Y_{i}-\pi_{i}\right) \mathrm{d} t+\sqrt{c Y_{i}} \mathrm{~d} W_{i}(t)-\sum_{j=1}^{N-1} Y_{i} \sqrt{c Y_{j}} \mathrm{~d} W_{j}(t), \quad i=1, \ldots, N
$$


of Gourieroux \& Jasiak [13] with $a<0, c>0, \pi_{\alpha}>0$, and $\sum_{j=1}^{N} \pi_{j}=1$.

In the univariate case, $K=N-1=1, \mathbf{Y}=\left(Y_{1}, Y_{2}\right)=(Y, 1-Y)$, the generalized Dirichlet, Dirichlet, Wright-Fisher, and Jacobi diffusions, Eqs. (2), (25), (26), (27), respectively, all reduce to

$$
\mathrm{d} Y(t)=\frac{b}{2}(S-Y) \mathrm{d} t+\sqrt{\kappa Y(1-Y)} \mathrm{d} W(t),
$$

see also [17], whose invariant is the beta distribution, which belongs to the family of Pearson diffusions, discussed in detail by Forman \& Sorensen [18].

\section{Summary}

Following the development in [2] we started with a multivariate distribution for a set of stochastic variables that satisfies a conservation principle in which all variables sum to unity. Applying the constraints on the existence of potential solutions of Fokker-Planck equations, we derived a system of stochastic differential equations (2) whose joint distribution in the statistically stationary state is Lochner's generalized Dirichlet distribution, Eq. (1). Eq. (2) is a generalization of the Dirichlet diffusion process developed in [2]. Compared to the standard Dirichlet process, the generalized diffusion allows for representing a more general class of stochastic processes with a more general covariance matrix. The process may be stationary or non-stationary, not limited to non-positive covariances, and satisfies the unit-sum requirement, Eq. (6), at all times, necessary for variables that obey a conservation principle.

\section{References}

[1] R. H. Lochner. A Generalized Dirichlet Distribution in Bayesian Life Testing. Journal of the Royal Statistical Society. Series B (Methodological), 37(1):pp. 103-113, 1975.

[2] J. Bakosi and J.R. Ristorcelli. A stochastic diffusion process for the Dirichlet distribution. Int. J. Stoch. Anal., 2013:7, 2013. Article ID 842981.

[3] R. J. Connor and J. E. Mosimann. Concepts of Independence for Proportions with a Generalization of the Dirichlet Distribution. J. Am. Stat. Assoc., 64(325):194-206, 1969.

[4] Tzu-Tsung Wong. Generalized Dirichlet distribution in Bayesian analysis. Appl. Math. Comput., $97(2-3): 165-181,1998$.

[5] N. L. Johnson. An approximation to the multinomial distribution some properties and applications. Biometrika, 47(1-2):93-102, 1960.

[6] J. E. Mosimann. On the compound multinomial distribution, the multivariate-distribution, and correlations among proportions. Biometrika, 49(1-2):65-82, 1962.

[7] S. Kotz, N.L. Johnson, and N. Balakrishnan. Continuous Multivariate Distributions: Models and applications. Wiley series in probability and statistics: Applied probability and statistics. Wiley, 2000.

[8] K. Pearson. Mathematical Contributions to the Theory of Evolution. On a Form of Spurious Correlation Which May Arise When Indices Are Used in the Measurement of Organs. Royal Society of London Proceedings Series I, 60:489-498, 1896.

[9] C.D.M. Paulino and de Braganca Pereira C.A. Bayesian methods for categorical data under informative general censoring. Biometrika, 82(2):439-446, 1995.

[10] F. Chayes. Numerical correlation and petrographic variation. J. Geol., 70:440-452, 1962. 
[11] P. S. Martin and J. E. Mosimann. Geochronology of pluvial lake Cochise, southern Arizona; [part] 3, Pollen statistics and Pleistocene metastability. Am. J. Sci., 263:313-358, 1965.

[12] K. Lange. Applications of the Dirichlet distribution to forensic match probabilities. Genetica, 96:107-117, 1995. 10.1007/BF01441156.

[13] C. Gourieroux and J. Jasiak. Multivariate Jacobi process with application to smooth transitions. Journal of Econometrics, 131:475-505, 2006.

[14] S. S. Girimaji. Assumed $\beta$-pdf model for turbulent mixing: validation and extension to multiple scalar mixing. Combust. Sci. Technol., 78(4):177 - 196, 1991.

[15] M. Steinrucken, Y.X. Rachel Wang, and Y.S. Song. An explicit transition density expansion for a multi-allelic Wright-Fisher diffusion with general diploid selection. Theoretical Population Biology, 83(0):1-14, 2013.

[16] C. W. Gardiner. Stochastic methods, A Handbook for the Natural and Social Sciences. SpringerVerlag, Berlin Heidelberg, 4 edition, 2009.

[17] J. Bakosi and J.R. Ristorcelli. Exploring the beta distribution in variable-density turbulent mixing. J. Turbul., 11(37):1-31, 2010.

[18] J. L. Forman and M. Sorensen. The Pearson Diffusions: A Class of Statistically Tractable Diffusion Processes. Scandinavian Journal of Statistics, 35:438-465, 2008.

[19] P. E. Kloeden and E. Platen. Numerical Solution of Stochastic Differential Equations. Springer, Berlin, 1999. 


\section{Appendix A: Inductive proof of Eqs. (14-16) based on $\mathrm{K}=3$}

Eqs. (14-16) are now arrived at for $K=3$, yielding the correspondence of the generalized Dirichlet distribution, Eq. (1), and its stochastic process, Eq. (2), for $K=3$. The procedure generalizes to arbitrary $K>3$.

From Eq. (11) the scalar potential for $K=3$ is

$$
\begin{aligned}
-\phi\left(Y_{1}, Y_{2}, Y_{3}\right)= & \left(\alpha_{1}-1\right) \ln Y_{1}+\left(\alpha_{2}-1\right) \ln Y_{2}+\left(\alpha_{3}-1\right) \ln Y_{3} \\
& +\gamma_{1} \ln \left(1-Y_{1}\right)+\gamma_{2} \ln \left(1-Y_{1}-Y_{2}\right)+\gamma_{3} \ln \left(1-Y_{1}-Y_{2}-Y_{3}\right) .
\end{aligned}
$$

From Eqs. (12-13) the drift and diffusion for $K=3$ are

$$
\begin{aligned}
a_{1}= & \frac{b_{1} / 2}{\left(1-Y_{1}\right)\left(1-Y_{1}-Y_{2}\right)}\left[S_{1}\left(1-Y_{1}-Y_{2}-Y_{3}\right)-\left(1-S_{1}\right) Y_{1}\right] \\
& \quad+\frac{Y_{1}\left(1-Y_{1}-Y_{2}-Y_{3}\right)}{\left(1-Y_{1}\right)\left(1-Y_{1}-Y_{2}\right)}\left[\frac{c_{11} / 2}{1-Y_{1}}+\frac{c_{12} / 2}{1-Y_{1}-Y_{2}}\right], \\
a_{2}= & \frac{b_{2} / 2}{1-Y_{1}-Y_{2}}\left[S_{2}\left(1-Y_{1}-Y_{2}-Y_{3}\right)-\left(1-S_{2}\right) Y_{2}\right]+\frac{c_{22}}{2} \cdot \frac{Y_{2}\left(1-Y_{1}-Y_{2}-Y_{3}\right)}{\left(1-Y_{1}-Y_{2}\right)^{2}}, \\
a_{3}= & \frac{b_{3}}{2}\left[S_{3}\left(1-Y_{1}-Y_{2}-Y_{3}\right)-\left(1-S_{3}\right) Y_{3}\right], \\
B_{11}= & \kappa_{1} \frac{Y_{1}\left(1-Y_{1}-Y_{2}-Y_{3}\right)}{\left(1-Y_{1}\right)\left(1-Y_{1}-Y_{2}\right)}, \\
B_{22}= & \kappa_{2} \frac{Y_{2}\left(1-Y_{1}-Y_{2}-Y_{3}\right)}{1-Y_{1}-Y_{2}}, \\
B_{33}= & \kappa_{3} Y_{3}\left(1-Y_{1}-Y_{2}-Y_{3}\right), \\
B_{12}= & B_{23}=B_{13}=0,
\end{aligned}
$$

Substituting Eqs. (29-36) into Eq. (9) for $K=3$ yields

$$
\begin{aligned}
& \frac{\alpha_{1}-1}{Y_{1}}-\frac{\gamma_{1}}{1-Y_{1}}-\frac{\gamma_{2}}{1-Y_{1}-Y_{2}}-\frac{\gamma_{3}}{1-Y_{1}-Y_{2}-Y_{3}}= \\
& =\left(\frac{b_{1}}{\kappa_{1}} S_{1}-1\right) \frac{1}{Y_{1}}+\left(\frac{c_{11}}{\kappa_{1}}-1\right) \frac{1}{1-Y_{1}}+\left(\frac{c_{12}}{\kappa_{1}}-1\right) \frac{1}{1-Y_{1}-Y_{2}} \\
& +\left[1-\frac{b_{1}}{\kappa_{1}}\left(1-S_{1}\right)\right] \frac{1}{1-Y_{1}-Y_{2}-Y_{3}}, \\
& \frac{\alpha_{2}-1}{Y_{2}}-\frac{\gamma_{2}}{1-Y_{1}-Y_{2}}-\frac{\gamma_{3}}{1-Y_{1}-Y_{2}-Y_{3}}= \\
& =\left(\frac{b_{2}}{\kappa_{2}} S_{2}-1\right) \frac{1}{Y_{2}}+\left(\frac{c_{22}}{\kappa_{2}}-1\right) \frac{1}{1-Y_{1}-Y_{2}}+\left[1-\frac{b_{2}}{\kappa_{2}}\left(1-S_{2}\right)\right] \frac{1}{1-Y_{1}-Y_{2}-Y_{3}}, \\
& \frac{\alpha_{3}-1}{Y_{3}}-\frac{\gamma_{3}}{1-Y_{1}-Y_{2}-Y_{3}}=\left(\frac{b_{3}}{\kappa_{3}} S_{3}-1\right) \frac{1}{Y_{3}}+\left[1-\frac{b_{3}}{\kappa_{3}}\left(1-S_{3}\right)\right] \frac{1}{1-Y_{1}-Y_{2}-Y_{3}},
\end{aligned}
$$


which shows that if

$$
\begin{aligned}
\alpha_{1} & =\frac{b_{1}}{\kappa_{1}} S_{1}, \\
\alpha_{2} & =\frac{b_{2}}{\kappa_{2}} S_{2}, \\
\alpha_{3} & =\frac{b_{3}}{\kappa_{3}} S_{3}, \\
1-\gamma_{1} & =\frac{c_{11}}{\kappa_{1}}, \\
1-\gamma_{2} & =\frac{c_{12}}{\kappa_{1}}=\frac{c_{22}}{\kappa_{2}}, \\
1+\gamma_{3} & =\frac{b_{1}}{\kappa_{1}}\left(1-S_{1}\right)=\frac{b_{2}}{\kappa_{2}}\left(1-S_{2}\right)=\frac{b_{3}}{\kappa_{3}}\left(1-S_{3}\right),
\end{aligned}
$$

all hold, the invariant of Eq. (2) is Eq. (1) for $K=3$,

$$
\begin{aligned}
& \mathscr{G}\left(Y_{1}, Y_{2}, Y_{3}, \alpha_{1}, \alpha_{2}, \alpha_{3}, \beta_{1}, \beta_{2}, \beta_{3}\right)= \\
& \quad \frac{\Gamma\left(\alpha_{1}+\beta_{1}\right) \Gamma\left(\alpha_{2}+\beta_{2}\right) \Gamma\left(\alpha_{3}+\beta_{3}\right)}{\Gamma\left(\alpha_{1}\right) \Gamma\left(\beta_{1}\right) \Gamma\left(\alpha_{2}\right) \Gamma\left(\beta_{2}\right) \Gamma\left(\alpha_{3}\right) \Gamma\left(\beta_{3}\right)} \times \\
& \quad \times Y_{1}^{\alpha_{1}-1} Y_{2}^{\alpha_{2}-1} Y_{3}^{\alpha_{3}-1}\left(1-Y_{1}\right)^{\gamma_{1}}\left(1-Y_{1}-Y_{2}\right)^{\gamma_{2}}\left(1-Y_{1}-Y_{2}-Y_{3}\right)^{\gamma_{3}},
\end{aligned}
$$

with

$$
\gamma_{1}=\beta_{1}-\alpha_{2}-\beta_{2}, \quad \gamma_{2}=\beta_{2}-\alpha_{3}-\beta_{3}, \quad \gamma_{3}=\beta_{3}-1 .
$$

Eqs. (40-45) give the correspondence between the coefficients of the stochastic system, Eq. (2), and its invariant, Eq. (1), for $K=3$. With Eq. (47) the correspondence between the parameters of the joint probability density function (PDF), $\left(\alpha_{1}, \alpha_{2}, \alpha_{3}, \beta_{1}, \beta_{2}, \beta_{3}\right)$, and the coefficients of the stochastic system, $\left(b_{1}, b_{2}, b_{3}, S_{1}, S_{2}, S_{3}, \kappa_{1}, \kappa_{2}, \kappa_{3}, c_{11}, c_{12}, c_{22}\right)$, is also given.

It is straightforward to verify that setting $\gamma_{1}=\gamma_{2}=0$ in Eq. (46) yields the Dirichlet distribution, Eq. (17), for $K=3 \quad(N=4)$,

$$
\begin{aligned}
& \mathscr{D}\left(Y_{1}, Y_{2}, Y_{3}, \omega_{1}, \omega_{2}, \omega_{3}, \omega_{4}\right)= \\
& \quad \frac{\Gamma\left(\omega_{1}+\omega_{2}+\omega_{3}+\omega_{4}\right)}{\Gamma\left(\omega_{1}\right) \Gamma\left(\omega_{2}\right) \Gamma\left(\omega_{3}\right) \Gamma\left(\omega_{4}\right)} Y_{1}^{\omega_{1}-1} Y_{2}^{\omega_{2}-1} Y_{3}^{\omega_{3}-1}\left(1-Y_{1}-Y_{2}-Y_{3}\right)^{\omega_{4}-1}
\end{aligned}
$$

with

$$
\omega_{1}=\alpha_{1}, \quad \omega_{2}=\alpha_{2}, \quad \omega_{3}=\alpha_{3}, \quad \omega_{4}=\beta_{3} .
$$

Similarly, setting $c_{11} / \kappa_{1}=c_{12} / \kappa_{1}=c_{22} / \kappa_{2}=1$ in Eqs. (37-38) reduces to the system corresponding that of the Dirichlet case [2].

\section{Appendix B: Numerical simulation: The effect of the extra coefficient for $K=2$}

Numerical simulations are used to demonstrate the effect of the extra coefficient, $c_{11}$, compared to the standard Dirichlet case, given in [2].

The time-evolution of an ensemble of 10,000 particles has been numerically computed by integrating the system (7), with drift and diffusion (12-13), for $K=2$, i.e., $\left(Y_{1}, Y_{2}, Y_{3}=1-Y_{1}-Y_{2}\right)$,

$$
\begin{aligned}
\mathrm{d} Y_{1} & =\frac{b_{1} / 2}{1-Y_{1}}\left[S_{1} Y_{3}-\left(1-S_{1}\right) Y_{1}\right] \mathrm{d} t+\frac{Y_{1} Y_{3}}{1-Y_{1}} \cdot \frac{c_{11} / 2}{1-Y_{1}} \mathrm{~d} t+\sqrt{\kappa_{1} \frac{Y_{1} Y_{3}}{1-Y_{1}}} \mathrm{~d} W_{1}, \\
\mathrm{~d} Y_{2} & =\frac{b_{2}}{2}\left[S_{2} Y_{3}-\left(1-S_{2}\right) Y_{2}\right] \mathrm{d} t+\sqrt{\kappa_{2} Y_{2} Y_{3}} \mathrm{~d} W_{2}, \\
Y_{3} & =1-Y_{1}-Y_{2} .
\end{aligned}
$$


Tab. 1: Coefficients of Eqs. (50-52) and asymptotic moments for three simulation cases.

Asymptotic moments for $K=2$, see Eqs. (19-20)

$$
\begin{aligned}
\left\langle Y_{1}\right\rangle & =\frac{\alpha_{1}}{\alpha_{1}+\beta_{1}} \\
\left\langle Y_{2}\right\rangle & =\frac{\alpha_{2}}{\alpha_{2}+\beta_{2}} \cdot \frac{\alpha_{1}}{\alpha_{1}+\beta_{1}} \\
\left\langle y_{1}^{2}\right\rangle & =\left\langle Y_{1}\right\rangle\left(\frac{\alpha_{1}+1}{\alpha_{1}+\beta_{1}+1}-\left\langle Y_{1}\right\rangle\right) \\
\left\langle y_{2}^{2}\right\rangle & =\left\langle Y_{2}\right\rangle\left(\frac{\alpha_{2}+1}{\alpha_{2}+\beta_{2}+1} \cdot \frac{\alpha_{1}+1}{\alpha_{1}+\beta_{1}+1}-\left\langle Y_{2}\right\rangle\right) \\
\left\langle y_{1} y_{2}\right\rangle & =\left\langle Y_{2}\right\rangle\left(\frac{\alpha_{1}}{\alpha_{1}+\beta_{1}+1}-\left\langle Y_{1}\right\rangle\right)
\end{aligned}
$$

PDF parameters from the SDE coefficients, see Eqs. (14-16)

$$
\begin{aligned}
\alpha_{1} & =\frac{b_{1}}{\kappa_{1}} S_{1} \\
\alpha_{2} & =\frac{b_{2}}{\kappa_{2}} S_{2} \\
1-\gamma_{1} & =\frac{c_{11}}{\kappa_{1}} \\
1+\gamma_{2} & =\frac{b_{1}}{\kappa_{1}}\left(1-S_{1}\right)=\frac{b_{2}}{\kappa_{2}}\left(1-S_{2}\right) \\
\beta_{2} & =1+\gamma_{2}=\frac{b_{1}}{\kappa_{1}}\left(1-S_{1}\right)=\frac{b_{2}}{\kappa_{2}}\left(1-S_{2}\right) \\
\beta_{1} & =\gamma_{1}+\alpha_{2}+\beta_{2}=1-\frac{c_{11}}{\kappa_{1}}+\alpha_{2}+\beta_{2}
\end{aligned}
$$

Dirichlet SDE coefficients (common to all cases)

$$
\begin{array}{ll}
b_{1}=1 / 10 & b_{2}=3 / 2 \\
S_{1}=5 / 8 & S_{2}=2 / 5 \\
\kappa_{1}=1 / 80 & \kappa_{2}=3 / 10
\end{array}
$$

Generalized Dirichlet SDE coefficients

$$
\begin{array}{ll}
c_{11}=\kappa_{11}=1 / 80 & (\text { case } 1) \\
c_{11}=-1 / 80 & (\text { case } 2) \\
c_{11}=-1 / 4 & (\text { case } 3)
\end{array}
$$

SDE asymptotic moments for cases $1,2,3$

$$
\begin{aligned}
& c_{11}=\frac{1}{80} \quad c_{11}=-\frac{1}{80} \quad c_{11}=-\frac{1}{4} \\
& \begin{array}{lll}
\alpha_{1}=5 & \alpha_{1}=5 & \alpha_{1}=5
\end{array} \\
& \begin{array}{ccc}
\alpha_{2}=2 & \alpha_{2}=2 & \alpha_{2}=2
\end{array} \\
& \beta_{2}=3 \quad \beta_{2}=3 \quad \beta_{2}=3 \\
& \begin{array}{lll}
\beta_{1}=5 & \beta_{1}=7 & \beta_{1}=26
\end{array} \\
& \left\langle Y_{1}\right\rangle=\frac{1}{2} \quad\left\langle Y_{1}\right\rangle=\frac{5}{12} \quad\left\langle Y_{1}\right\rangle=\frac{5}{31} \\
& \left\langle Y_{2}\right\rangle=\frac{1}{5} \quad\left\langle Y_{2}\right\rangle=\frac{7}{30} \quad\left\langle Y_{2}\right\rangle=\frac{52}{155} \\
& \left\langle y_{1}^{2}\right\rangle=\frac{1}{44} \quad\left\langle y_{1}^{2}\right\rangle=\frac{35}{1872} \quad\left\langle y_{1}^{2}\right\rangle=\frac{65}{15376} \\
& \left\langle y_{2}^{2}\right\rangle=\frac{4}{275} \quad\left\langle y_{2}^{2}\right\rangle=\frac{609}{35100} \quad\left\langle y_{2}^{2}\right\rangle=\frac{11141}{384400} \\
& \left\langle y_{1} y_{2}\right\rangle=-\frac{1}{110} \quad\left\langle y_{1} y_{2}\right\rangle=-\frac{35}{4680} \quad\left\langle y_{1} y_{2}\right\rangle=-\frac{13}{7688}
\end{aligned}
$$

In Eqs. (50-51) $\mathrm{d} W_{1}$ and $\mathrm{d} W_{2}$ are independent Wiener processes, sampled from Gaussian streams of random numbers with mean $\left\langle\mathrm{d} W_{i}\right\rangle=0$ and covariance $\left\langle\mathrm{d} W_{i} \mathrm{~d} W_{j}\right\rangle=\delta_{i j} \mathrm{~d} t$. Eqs. (50-52) were advanced in time with the Euler-Maruyama scheme [19] with time step $\Delta t=0.025$. The coefficients of the stochastic system (50-52), the corresponding parameters and the first two moments of the asymptotic generalized Dirichlet distributions for $K=2$ are shown in Table 1. Three different cases were simulated. Here the initial condition of $\left(Y_{1}, Y_{2}\right) \equiv 0$ was used. The initial PDF in all cases is the same: all samples are zero and the PDF is therefore not Dirichlet nor Generalized Dirichlet, see also [2] for nonzero but different non-Dirichlet initial conditions. Our motivation is two-fold: (1) to show that the solution approaches the invariant, and (2) to show how the new additional parameter in the generalized Dirichlet SDE affects the dynamics. Had the initial conditions coincided with the given invariant, the PDF (and its statistics) would not have changed in time - as has been 

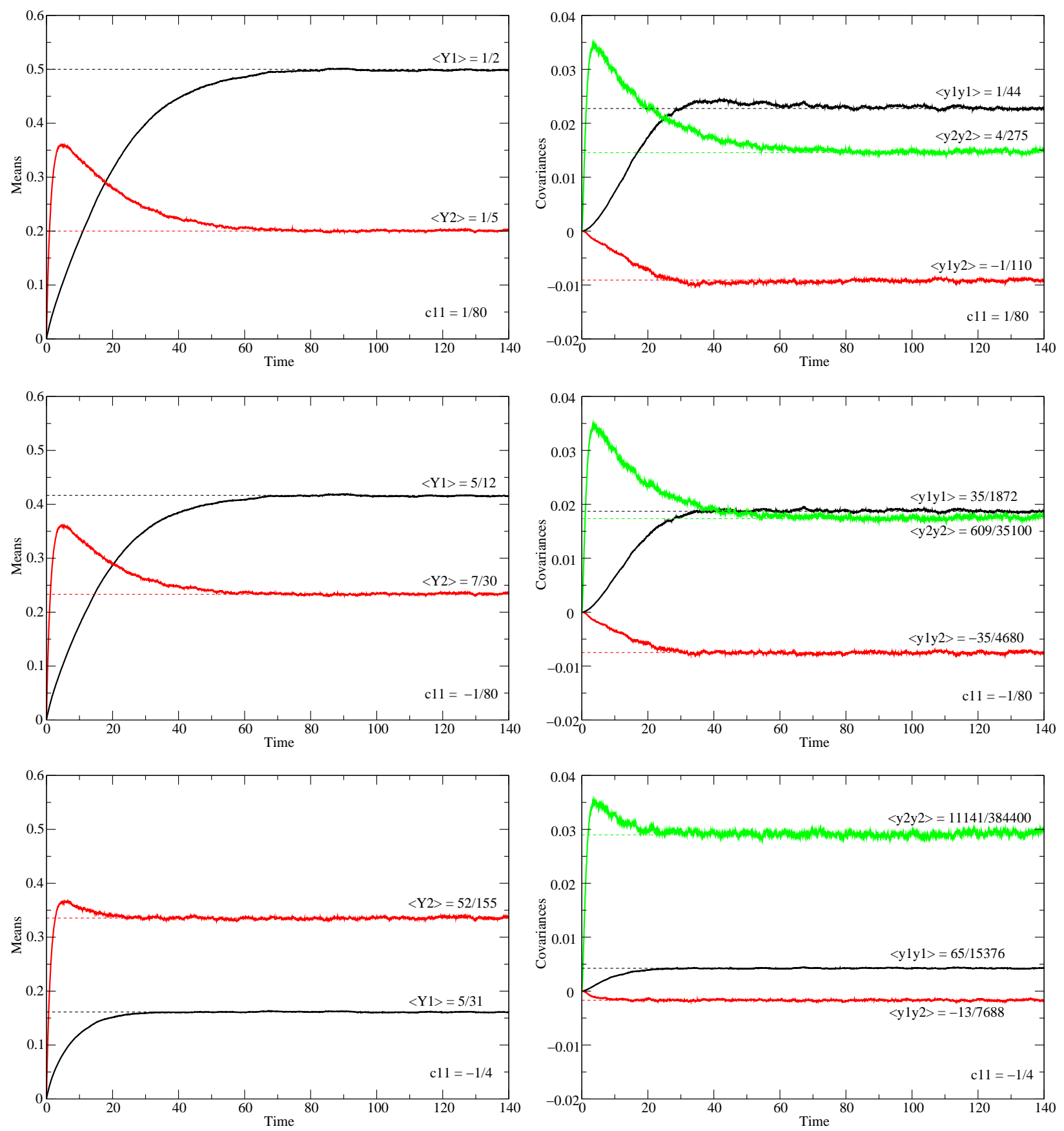

Fig. 1: Time evolution of the first two moments of Eqs. (50-52). First row: $c_{11}=\kappa_{1}=1 / 80$ (standard Dirichlet, see also [2]), second row: $c_{11}=-1 / 80$, third row: $c_{11}=-1 / 4$.

demonstrated mathematically. The SDE coefficients in the three simulations only differ in the extra generalized Dirichlet coefficient, $c_{11}$, otherwise, the setup corresponds to the example in [2]. In the first simulation $c_{11}=\kappa_{1}=1 / 80$, i.e., $c_{11}$ is not a free coefficient and is chosen to yield an asymptotic solution that is a (standard) Dirichlet, the same as in [2]. In the second and third simulations $c_{11}$ are freely chosen and thus yield generalized Dirichlet solutions. Figure 1 shows the evolutions of the first two moments in time for the three cases. 